\section{Increased Screen Time - A Pandemic Era Trigger for Neuro-Cardiogenic Syncope}

The COVID-19 pandemic has resulted in a revamp of our everyday life, which is being dubbed as the new normal. As people get adjusted to work and study from home, there are new challenges in maintaining physical and mental health. An unusual pattern noted by the author is an increase in the number of children diagnosed with autonomic syncope in recent months. Between August and November, 2020, 16 children were diagnosed with neuro-cardiogenic syncope in the author's clinic, as compared to only three children during the same period in 2019. The age group of these children varied between 7-16 years. All children were subject to a detailed history regarding the event as well as potential red flag signs [1]. All episodes happened during the daytime and when the children were either seated or standing. None of the episodes happened during an exertion; although, in one child, there were three episodes that happened immediately after an exertion. Almost all episodes were preceded by a prodromal symptom which included light headedness, aura and/or vertigo. There was a prompt and complete recovery and there were no injuries. The children presented for evaluation after a median (range) time of 2 days ( 4 hours -7 days) after the event. They underwent recording of postural vital signs and a standardized 12 lead electrocardiogram.

When enquired, each of the parents revealed a concern about increased daily screen time on smart devices for their children. The older children spent more time on the screen due to a cramped schedule of online classes while in the younger children, smartphone use replaced other forms of physical activity curtailed by COVID-induced lockdowns. The family were counselled about lifestyle modifications including sleep hygiene, adequate water and salt intake and reduction of screen time. Further evaluation for autonomic syncope was not carried out. All parents were contacted three to six months after their presentation to assess the efficacy of the intervention. The parents reported compliance with the suggested lifestyle measures and no recurrence of syncope during the short period of follow-up.

Neuro-cardiogenic syncope is common in children with 1 in 6 children reported to have at least one episode before adulthood [2]. It is possible that the increased screen time and exposure to high-definition screens results in increased eye strain and fatigue which reduces the threshold for fainting. In particular, high color temperature display and display flickering have been shown to cause eye fatigue [3]. Dizziness and pre-syncope have previously been reported to be potential adverse events of smartphone use, and syncope is likely an extension of the same pathophysiological process [4].

The findings are based on personal observations in clinical practice and highlight the importance of a detailed history in evaluation of syncope. Prospective studies with assessment of multiple associated factors of syncope may elucidate this issue further. However, till that time, pediatricians should continue to counsel the parents about the importance of limiting screen time in children.

Mani RaM Krishna Dr RK Hospital for Women and Children, Thanjavur, Tamil Nadu, India. mann_comp@hotmail.com

\section{REFERENCES}

1. Krishna MR, Kunde MF. A clinical approach to syncope. Indian J Pract Pediatr. 2020;22:92-9.

2. McHarg ML, Shinnar S, Rascoff H, Walsh CA. Syncope in childhood. Pediatr Cardiol. 1997;18:367-71.

3. Seltman W. Prevent eyestrain from digital devices [Internet] WebMD LLC; c2005-2021. [updated 2019 August 6]. Available from https://www.webmd.com/eye-health/preventdigital-eyestrain.

4. Al-Khlaiwi T, Meo SA. Association of mobile phone radiation with fatigue, headache, dizziness, tension and sleep disturbance in Saudi population. Saudi Med J. 2004;25:732-6.

\section{Should High Flow Nasal Cannula Therapy Be the Primary Mode of Respiratory Support in a Pediatric Intensive Care Unit? Questions Remain!}

We read with interest the recent paper on high flow nasal cannula (HFNC) therapy as a primary mode of respiratory support in a pediatric intensive care unit (PICU) [1]. To better understand the paper, we request response to the following queries: i) As the aim of the study was to assess the efficacy of HFNC as the primary mode of respiratory support in PICU, we think that a cross sectional study design was inappropriate. Instead, a randomized controlled trial (RCT) study design, if used, would have given clearer answers. In the RCT, other modes of non-invasive ventilation (NIV) could have been used as the comparator and the primary outcome measured could have been the percentage of children going on to invasive mechanical ventilation.

ii) The authors mention targeting oxygen concentration between $92-97 \%$ for all children between 1 month to 16 years? Is there a validated reference or protocol for adjusting $\mathrm{FiO}_{2}$ to target such a saturation up to $97 \%$ ? 